\title{
Intercomparison of the LASCO-C2, SECCHI-COR1, SECCHI-COR2, and Mk4 Coronagraphs
}

\author{
Richard A. Frazin • Alberto M. Vásquez • William T. Thompson • Russell J. Hewett • \\ Philippe Lamy • Antoine Llebaria • Angelos Vourlidas • Joan Burkepile
}

Received: 21 July 2011 / Accepted: 8 May 2012 / Published online: 8 June 2012

(C) Springer Science+Business Media B.V. 2012

\begin{abstract}
In order to assess the reliability and consistency of white-light coronagraph measurements, we report on quantitative comparisons between polarized brightness $[p B]$ and total brightness $[B]$ images taken by the following white-light coronagraphs: LASCO-C2 on SOHO, SECCHI-COR1 and -COR2 on STEREO, and the ground-based MLSO-Mk4.
\end{abstract}

Now R.J. Hewelt at Dept. of Mathematics, Massachusetts Institute of Technology, Cambridge, MA, 02139, USA

R.A. Frazin $(\bowtie)$

Dept. of Atmospheric, Oceanic and Space Sciences, University of Michigan, Ann Arbor, MI 48109, USA

e-mail: rfrazin@umich.edu

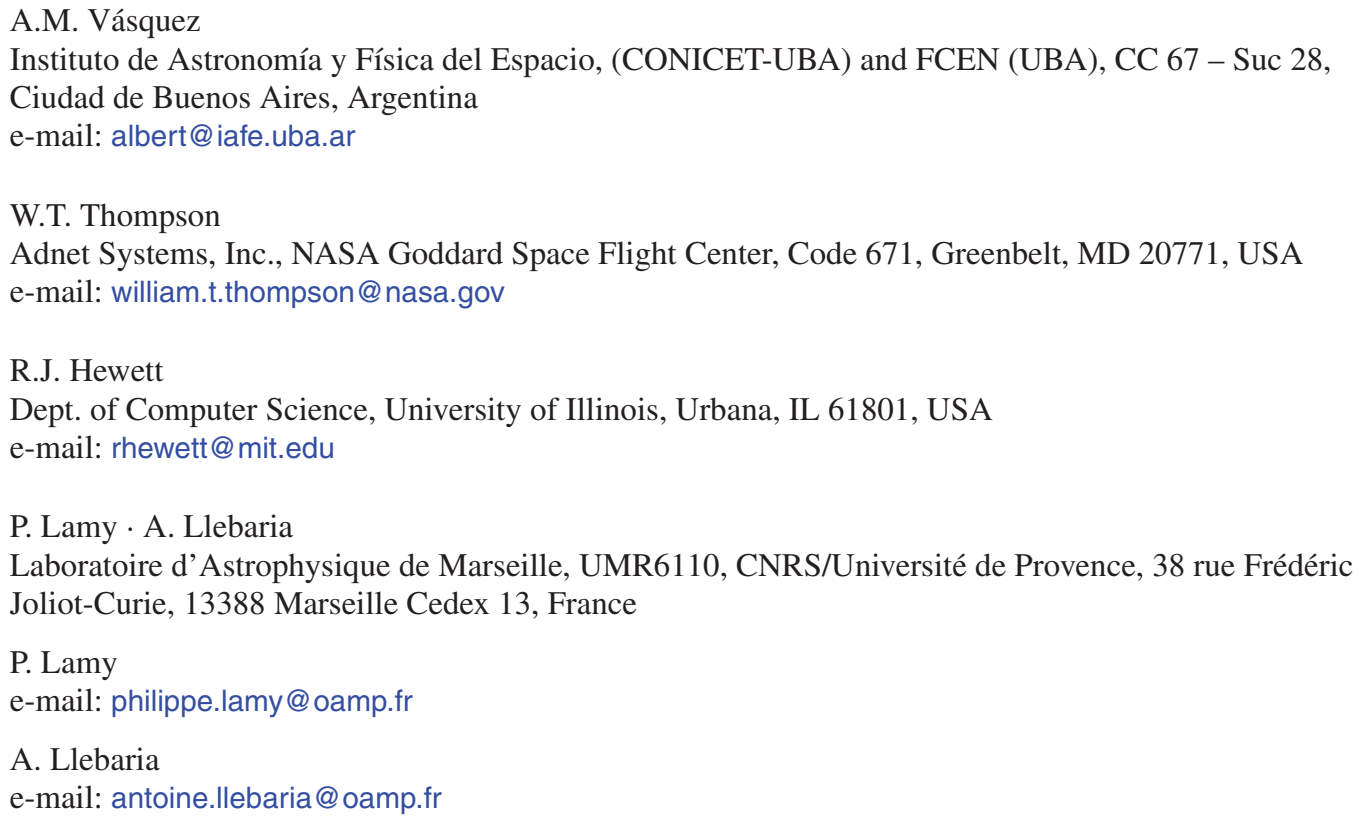


The data for this comparison were taken on 16 April 2007, when both STEREO spacecraft were within $3.1^{\circ}$ of Earth's heliographic longitude, affording essentially the same view of the Sun for all of the instruments. Due to the difficulties of estimating stray-light backgrounds in COR1 and COR2, only Mk4 and C2 produce reliable coronal-hole values (but not at overlapping heights), and these cannot be validated without rocket flights or groundbased eclipse measurements. Generally, the agreement between all of the instruments' $p B$ values is within the uncertainties in bright streamer structures, implying that measurements of bright CMEs also should be trustworthy. Dominant sources of uncertainty and stray light are discussed, as is the design of future coronagraphs from the perspective of the experiences with these instruments.

Keywords Coronagraph $\cdot$ Electron density $\cdot$ Solar corona

\section{Introduction}

White-light measurements of the K-corona's polarized brightness $[p B]$ and total brightness $[B]$ are particularly valuable, since they are direct diagnostics of the electron density (van de Hulst, 1950). Empirical knowledge of the electron density is critical for model validation (Jin et al., 2012) and for understanding the propagation of coronal mass ejections (CMEs; Zuccarello et al., 2012), to name a few applications. White-light coronagraphs (Lyot, 1939) are the only means to measure the coronal optical emission, except during eclipses (Frost, 1900); hence, it is important to assess their performance. These measurements are notoriously difficult in regions of low density (e.g. coronal holes) due to stray light and sky background (for ground-based instruments).

This article compares the $p B$ and $B$ measurements from six coronagraphs taken on 16 April 2007: the Mauna Loa Solar Observatory Mk4 ( $p B$ only), Solar and Heliospheric Observatory (SOHO)/Large Angle and Spectrometric Coronagraph (LASCO)-C2 (Brueckner et al., 1995), and Solar Terrestrial Relations Observatory (STEREO)/Sun Earth Connection Coronal and Heliospheric Investigation (SECCHI)-COR1 and -COR2 (Howard et al., 2008). LASCO-C3 is not included in this discussion because one of the polarizers rapidly degraded after the 1998 mission interruption. In addition, there may be significant F-corona polarization in the $\mathrm{C} 3$ height range that makes $\mathrm{F}$ - and K-corona separation difficult (Hayes, Vourlidas, and Howard, 2001). The intercalibration team, which includes the authors of this article, maintains a website to document the progress of calibration/intercalibration issues: secchi-ical.wikidot.com/.

The literature on coronagraph intercalibration is quite limited. Electron densities from the van de Hulst (1950) inversion of the Mk4 data were compared to those derived from Ultraviolet Coronagraph Spectrometer (UVCS: Kohl et al., 1995) measurements of the O VI 1032, 1036.7 A doublet by Lee et al. (2008), finding rough agreement. Frazin et al. (2002) compared the $\mathrm{C} 2 \mathrm{pB}$ measurements (preliminary calibration, see below) to those made with the well-characterized, single-pixel UVCS White Light Channel (WLC: Romoli et al., 2002).

\footnotetext{
A. Vourlidas

Naval Research Laboratory, Code 7663, Washington, DC 20375, USA

J. Burkepile

High Altitude Observatory, National Center for Atmospheric Research, P.O. Box 3000, Boulder, CO 80307, USA

e-mail: iguana@ucar.edu 
(The final calibration of $\mathrm{C} 2$, described below, is likely to improve the agreement with the UVCS WLC.) Previous comparisons of COR1 to other coronagraphs are cited below.

Elmore et al. (2003) discuss the calibration of Mk4. The radiometric and pointing calibration of COR1 is described by Thompson and Reginald (2008), who also compare CME $p B$ values of COR1 to Mk4 and $\mathrm{C} 2$. That study found COR1-A and -B radiometric calibrations to be indistinguishable from each other but $\approx 20 \%$ greater than that of $\mathrm{C} 2$ (preliminary calibration, see below), meaning that the CME was determined to have a higher intrinsic brightness in COR1. Thompson et al. (2010) discuss procedures for determining the straylight background in COR1 and compare the background-subtracted images to those of Mk4, producing results consistent with those given in this article. Thompson et al. (2011) took advantage of the 6 February 2011 nearly perfect opposition $\left(\approx 180^{\circ}\right.$ separation) of the two STEREO spacecraft to compare the COR1-A and -B images, which ideally should be perfect mirror images of each other, and found excellent agreement between them.

The dominant source of COR1 background is dust on the front and back surfaces of the objective lens, resulting in backgrounds that are $\approx 25$ times brighter than streamers near $1.6 R_{\odot}$, increasing by about an order of magnitude as one approaches $4 R_{\odot}$. From launch until 30 January 2009, when a dust particle migrated to the COR1-B objective, COR1-B was substantially cleaner than COR1-A (although both were well within design specifications). Since that date, the level of scattering in COR1-A and -B has been comparable.

The preliminary calibration of $\mathrm{C} 2$ was performed by the Naval Research Laboratory (NRL) near the beginning of the SOHO mission. The images processed with the preliminary calibration are publicly available on the NRL C2 $p B$ archive: lasco-www.nrl.navy.mil/ content/retrieve/polarize/. During the subsequent years, the Laboratoire d'Astrophysique de Marseille (LAM) team has developed sophisticated calibration and F-corona removal procedures that result in a $\mathrm{C} 2$ calibration for both $p B$ and $B$ that is significantly different from the preliminary calibration (Llebaria, Lamy, and Danjard, 2006). The final radiometric calibration of C2 performed by LAM has been published by Llebaria, Lamy, and Danjard (2006), and Llebaria, Loirat, and Lamy (2010) provide a procedure for removing the F-corona component from its $B$ images (see also Morgan and Habbal, 2007). The polarimetric calibration of C2 and C3 is discussed by Moran et al. (2006), but their procedures have been superseded by the work of the LAM team. Comparing the initial and final $p B$ calibrations, one finds significant differences with complex spatial structure, including the following:

- The ratio image, i.e. the pixel-by-pixel values of [preliminary/final], exhibits an " $\mathrm{X}$ " pattern due to retardance introduced by the folding mirrors that was not corrected in the preliminary calibration.

- The average ratio, taken over the useful field of view, [preliminary/final] $\approx 1.50$.

- The asymmetry between the North and South Polar coronal holes seen in the initial calibration has been removed.

The final calibration is the subject of a forthcoming publication by the LAM team.

The dominant source of stray light in COR2 is light from the solar disk diffracted by the external occulter that is not blocked by the internal occulter due to a post-launch misalignment between the two occulters. The cause of the misalignment cannot be determined with certainty, but mechanical stress during launch is the most likely explanation. The degree of misalignment (and hence the stray-light levels) is more severe for COR2-B, which prevents the detection of faint coronal structures around the southern rim of the occulter up to a distance of $\approx 4.1 R_{\odot}$ (in the North, the corona can be observed above $\approx 3 R_{\odot}$ ). While the absolute stray-light levels diminish with increasing distance from the occulter, the ratio of coronal signal to scattered-light background is $\approx 1-2$ between $\approx 5-8 R_{\odot}$, rising to $\approx 25$ 
around the occulter $\left(\approx 3 R_{\odot}\right)$, and rising again to $\approx 40$ at $15 R_{\odot}$. Details on the on-orbit performance of the COR2 coronagraphs will be reported in an upcoming article.

Section 2 explains the instrumental uncertainties. Section 3 first describes the observations, and then discusses the instrument comparisons, one pair at a time. Section 4 draws conclusions based on the work presented here, including lessons that should be considered when designing future coronagraphs.

\section{Uncertainty Estimates}

This intercalibration effort utilizes the best-known estimates of the uncertainties for each of the instruments, and this information is reflected in error bars $( \pm 1 \sigma)$ in the plots for the individual comparisons shown below. While all photometric measurements have shot noise, it is not a significant contribution to any of the measurements here, as other sources of uncertainty are dominant. For COR1-A, -B, and COR2-B, the large stray-light background can make the shot noise important when considering single pixels. Here, this component of the noise has been much suppressed with the spatial and temporal binning described below. In some cases, the uncertainties have unknown components, and the error bars only reflect the known components. The error bars for each instrument are described here.

- The Mk4 uncertainty of $\pm 3 \times 10^{-9} B_{\odot}$ corresponds to uncorrected sky polarization and detector bit error, which throws away significant bits. This error is newly discovered.

- The radiometric uncertainty (derived from Jupiter brightness measurements) of COR1 is estimated to be $\pm 10 \%$ (Thompson and Reginald, 2008). The temporal variation in the background intensity is characterized by $\pm 1 \times 10^{-10} B_{\odot}$. We take the total COR 1 uncertainty to be the quadrature sum of these two quantities. This is an underestimate, as it ignores the systematic error caused by using the /calroll background, which results in a global underestimate of the brightness (the near-zero values of the coronal holes are a symptom of this). This systematic error has been evaluated by comparisons to Mk4 (see Table 2 in Thompson et al., 2010), but is not included in the error bars shown here, since we desire an independent validation insofar as possible.

- The dominant sources of uncertainty in C2 are not easy to identify, as the error analysis is complex and requires Monte-Carlo error propagation. In the end, the $C 2 p B$ and $B$ uncertainties are likely to be dominated by uncorrected polarization arising from the fold mirrors and the imperfections of the three polaroid foils, which deviate substantially from the ideal. The resulting uncertainty maps have some local variation, but are reasonably described by a global value of $\pm 15 \%$ of the measured $p B$ and $B$ values.

- The radiometric uncertainty of COR2 is thought to be $\pm 20 \%$, based on pre-flight measurements and comparisons of CME masses (Colaninno and Vourlidas, 2009) calculated in $\mathrm{C} 2$ and COR2, and this is the value adopted here. Since the background uncertainties have not been estimated, this error bar represents a lower bound on the total uncertainty.

\section{Comparisons}

Although SOHO, STEREO-A and -B, and the Earth saw almost exactly the same face of the Sun from STEREO's launch in late 2006 through January 2007, the STEREO pointing was 
not finalized until the end of February 2007. ${ }^{1}$ Performing the comparison with STEREO's final pointing is critical, since the pointing has a major influence on the stray-light properties of COR1 and COR2. The time chosen for this comparison is therefore 16 April 2007, about seven weeks after the final STEREO pointing was established. This allows enough time for the monthly background estimates to adjust to the new pointing. Using similar reasoning, Thompson et al. (2010) compared COR1 to Mk4 using data from 2 April 2007. The backgrounds in the STEREO-B coronagraphs are likely to be somewhat compromised by the spacecraft's rotation throughout the month of April. This is probably more important for COR2 than COR1, because the F-corona is more symmetric at low heights.

LASCO-C2 took one $p B$ sequence on 16 April 2007, at 20:57 UT. The C2 image processed with the preliminary calibration will henceforth be called C2-P. For this article, the LAM team processed the same $p B$ sequence, and this final result will henceforth be called $\mathrm{C} 2-\mathrm{F}$. In this article, $\mathrm{C} 2-\mathrm{F}$ is compared to $\mathrm{Mk} 4, \mathrm{COR} 1$, and COR2, and C2-P is not considered further. No binning or other post-processing was applied to the $\mathrm{C} 2$ images, resulting in a format of $512 \times 512$ pixels, as is standard for $\mathrm{C} 2 \mathrm{pB}$ sequences, each pixel projecting to $(23.8 \mathrm{arcsec})^{2}$ on the corona. The sequences of polarized images yield both $p B$ and $B$ values. An extensive effort has been made by the LAM team to model the F-corona and remove it from the C2 B images. This process is described briefly by Frazin et al. (2010), who also present the first quantitative tomography from $B$ images, yielding three-dimensional electron densities. Below we will show comparisons of the $C 2 B$ and $p B$ images based on the final calibration to those made by the other instruments.

The Mk4 image is the standard daily "average" image available on the Mauna Loa Solar Observatory (MLSO) website: mlso.hao.ucar.edu. This $p B$ image is an average over a 50-minute period ending at 17:55 UT. The (rectangular format) MLSO image was spatially binned $4 \times 4$ resulting in an effective pixel size of $(23.9 \operatorname{arcsec})^{2}$.

COR1 on the STEREO-A and -B spacecraft took $p B$ sequences every ten minutes throughout the entire day. For the comparison to Mk4, seven COR1 $p B$ sequences, spanning one hour and centered on 17:30 were chosen. The comparison between $\mathrm{C} 2$ and COR1 also used seven $p B$ sequences spanning one hour, but centered at 21:00. The COR1 images made from the sequences at these two different times are nearly identical. The COR1 images were processed with the secchi_prep.pro routine, ${ }^{2}$ setting the /calroll (and /interpolate) flag, to subtract background images that combine information from the instrument calibration rolls and the monthly minimum values (Thompson et al., 2010). The /calroll backgrounds, which combine information from spacecraft rolls and monthly minimum procedures, essentially take the coronal-hole signal to be background. As shown below, this appears to be adequate for bright structures such as streamers, but it forces the coronal hole values to zero. Using the background-subtracted sequences, the cor1_fitpol.pro routine was used to produce the $p B$ and $B$ images. The resulting $1024 \times 1024$ COR 1 images were spatially binned $4 \times 4$ and temporally binned over about one hour, which consisted of averaging the seven $p B$ images for both A and B. This spatial binning results in an effective pixel size of $(30.0 \mathrm{arcsec})^{2}$.

We selected three COR2 $p B$ sequences centered on 20:53, and spanning one hour (30-minute cadence). They were averaged to create the $p B$ and $B$ images used for this analysis. Similarly to the COR1 procedure to produce the $p B$ images, each $p B$ sequence was

\footnotetext{
${ }^{1}$ The repointings of both STEREO spacecraft in February 2007 were performed to reduce the stray light caused by internal misalignments in COR2-A and -B. These repointings changed the spatial distribution of stray light in COR1-A and -B, but they did not increase the overall amount of stray light.

${ }^{2}$ All routines, which have a .pro suffix, are written in the IDL language and are freely available in the SolarSoft package: www.Imsal.com/solarsoft/.
} 
Figure 1 An enhanced composite of three simultaneously taken images of the corona on 16 April 2007 (Morgan, Habbal, and Woo, 2006). From the center to the outer part: i) EIT $304 \AA$ band, ii) $\mathrm{Mk} 4$, and iii) $\mathrm{C} 2$ (unpolarized).

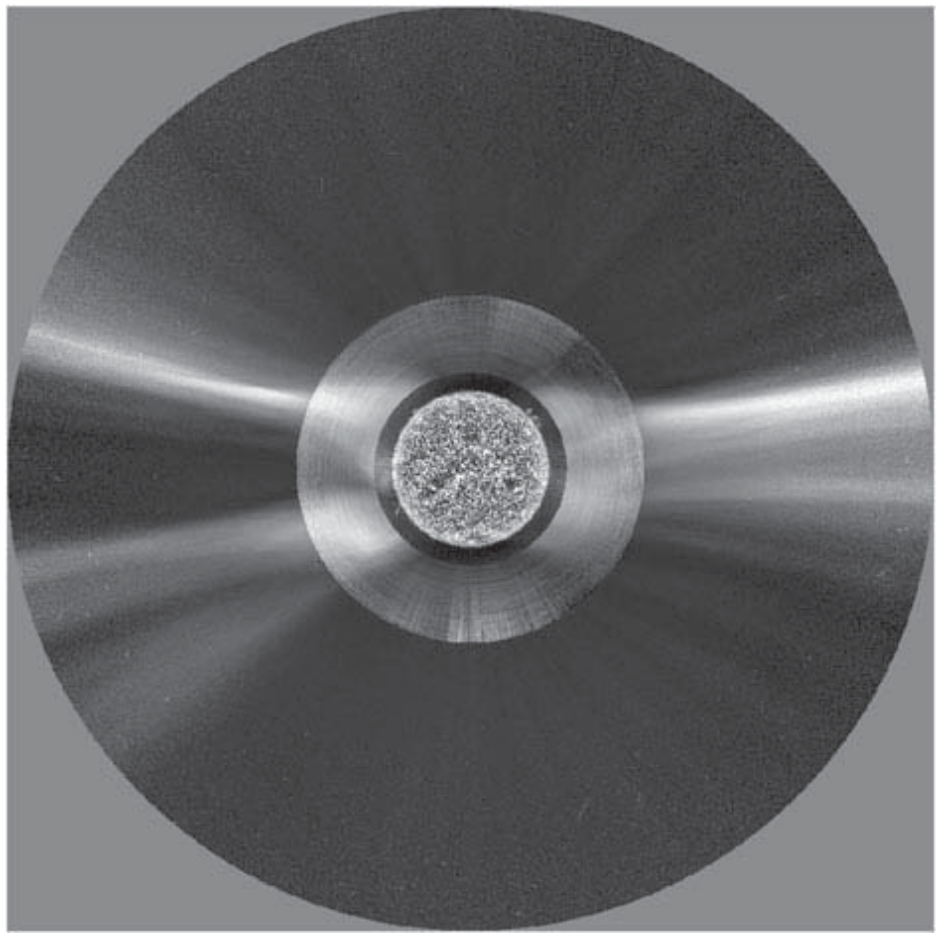

Table 1 Apparent size of the solar radius and approximate "useful" fields of view of the coronagraphs (defined by $R_{\min }$ and $\left.R_{\max }\right)$. The last two columns give the respective Carrington latitudes and longitudes on 16 April 2007 at 21:00 UT.

\begin{tabular}{lllcll}
\hline Instrument & $\begin{array}{l}R_{\odot} \\
{\left[{ }^{\prime \prime}\right]}\end{array}$ & $\begin{array}{l}R_{\min } \\
{\left[R_{\odot}\right]}\end{array}$ & $\begin{array}{l}R_{\max } \\
{\left[R_{\odot}\right]}\end{array}$ & $\begin{array}{l}\text { Latitude } \\
{\left[{ }^{\circ}\right]}\end{array}$ & $\begin{array}{l}\text { Longitude } \\
{\left[{ }^{\circ}\right]}\end{array}$ \\
\hline Mk4 & 956.3 & 1.15 & 2.5 & -5.50 & 143.65 \\
C2 & 966.1 & 2.2 & 6.1 & -5.48 & 143.79 \\
COR1-A & 996.5 & 1.45 & 3.9 & -5.23 & 146.89 \\
COR1-B & 928.0 & 1.55 & 4.0 & -5.82 & 142.42 \\
COR2-A & 996.5 & 3.0 & 15.0 & -5.23 & 146.89 \\
COR2-B & 928.0 & 4.1 & 16.0 & -5.82 & 142.42 \\
\hline
\end{tabular}

processed with background subtraction for each polarizer angle. No /calroll backgrounds are presently available for COR2, and monthly minimum backgrounds were used instead. The background images were obtained using the scc_getbkgimg.pro routine, and then subtracted from the output of the secchi_prep.pro routine, without the /calroll flag. Using the background-subtracted sequences, the cor_polariz.pro routine produced the $p B$ and $B$ images. A second set of $B$ images was produced with the standard procedure: applying secchi_prep.pro with the /polariz_on flag, and then subtracting a total background image, also downloaded with scc_getbkgimg.pro. The $B$ images produced using the two procedures were almost identical, with a slight improvement when using the standard procedure (used for the present analysis). The COR 2 images were binned $2 \times 2$, resulting in an effective pixel size of $(29.4 \mathrm{arcsec})^{2}$.

Figure 1 shows an image of the corona on 16 April 2007 enhanced with the method developed by Morgan, Habbal, and Woo (2006). The corona is dominated by two streamers on the east limb and one on the west. These streamers provide the best opportunity to obtain reliable $p B$ values, since their large intensities make the background subtraction less important. Figure 2 displays the coronagraph images used for this intercalibration study, and Table 1 summarizes the Carrington heliographic coordinates (Thompson, 2006) and fields 


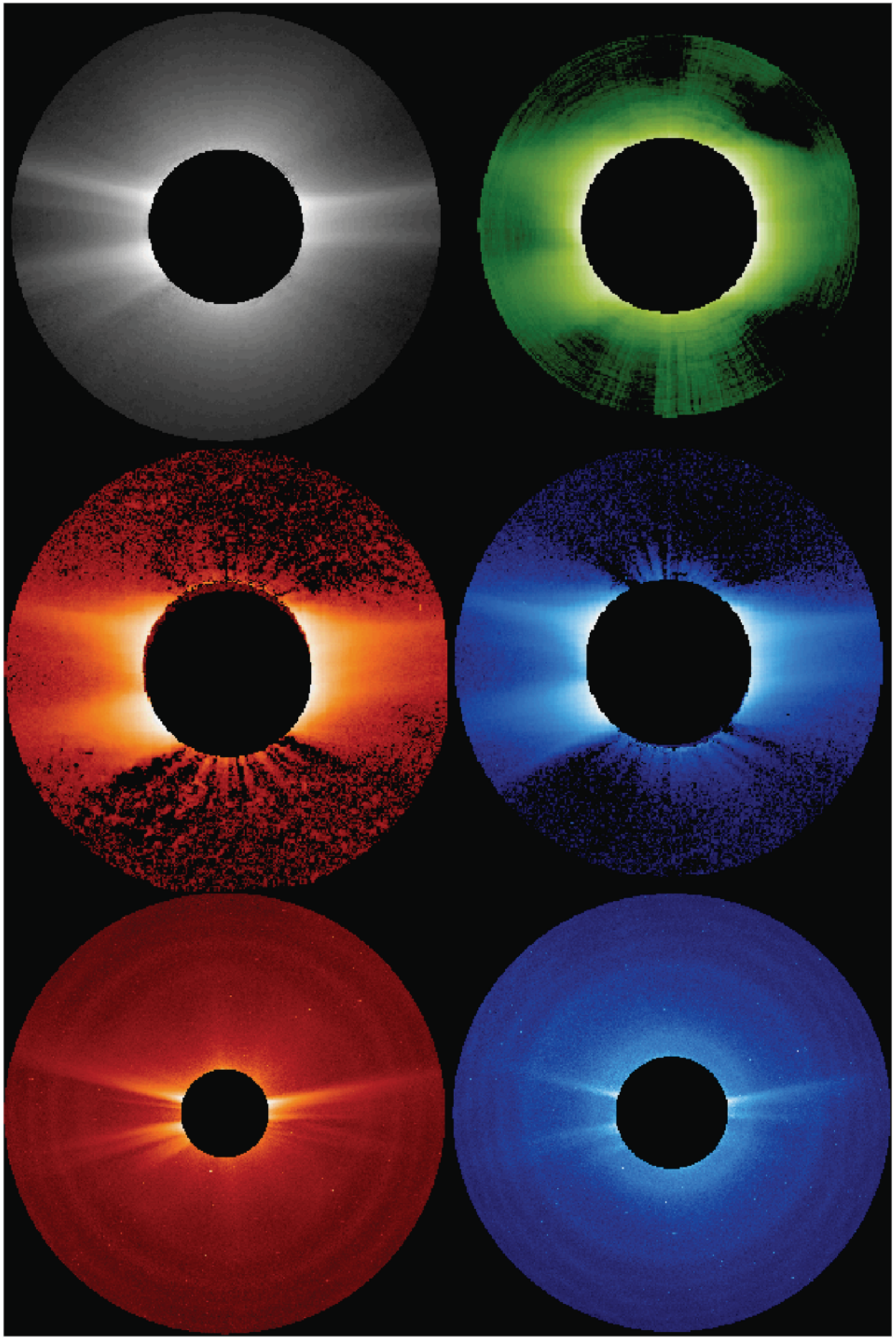

Figure 2 The coronagraph $p B$ images used for the intercomparisons in this study, shown in logarithmic scale. Upper left: C2, upper right: Mk4, middle left: COR1-A, middle right: COR1-B, lower left: COR2-A, lower right: COR2-B. The respective fields of view fields-of-view are detailed in Table 1. A processed version of the Mk4 image used for this comparison can be seen in Figure 1. 
Figure 3 The images of the ratio of the polarized brightness $[p B]$ COR1/Mk4 for COR1-A (top) and COR1-B (bottom). The white pixels correspond to values greater than the limits of the color scale. The radius of the blocked portion is $1.5 R \odot$, and the overlaid grid starts at $1.5 R_{\odot}$, has circles every $0.5 R \odot$, and radial lines every $30^{\circ}$.
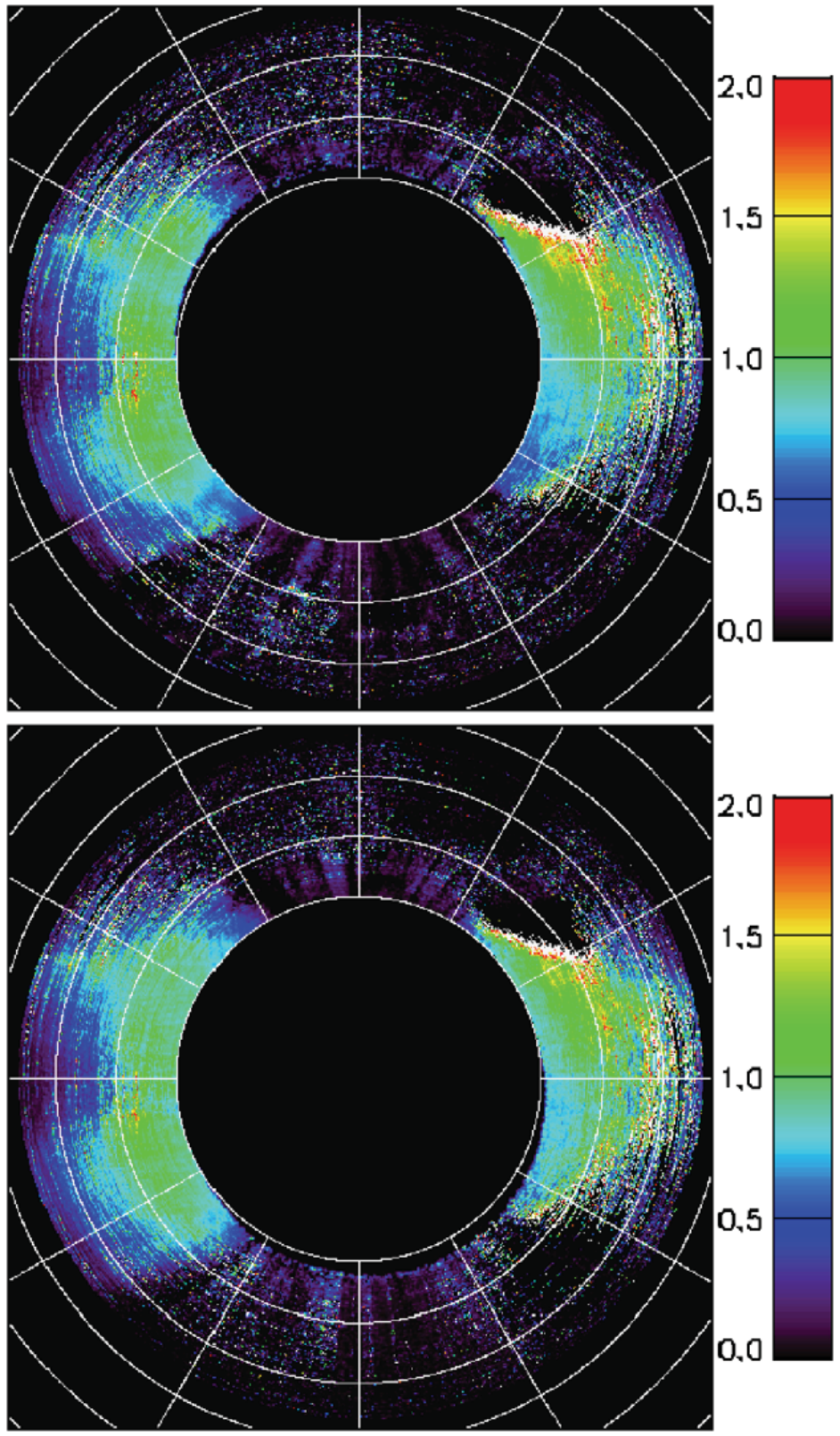

of view of the coronagraphs on 16 April 2007 at 21:00 UT. The observable inner radius and the outer radius of each image are given in Table 1 . These radii were chosen to maximize the amount of possibly useful data and represent fields of view that are larger than would be optimal for accurate measurements.

To evaluate the importance of projection effects (i.e. changes due to the differences in the heliographic longitudes of the various coronagraphs), we repeated some of the analyses given below, but using time delays in COR 1 and COR2 data selection to take advantage of the Sun's rotation so that the corona is seen from approximately the same angle in Carrington coordinates (Thompson, 2006). The time differences are relatively small (2.32 hours before for STEREO-B and 5.92 hours after for STEREO-A). Despite the temporal evolution of the streamers during this period, the analysis of these time-shifted observations yielded results that are nearly indistinguishable from those presented here. 

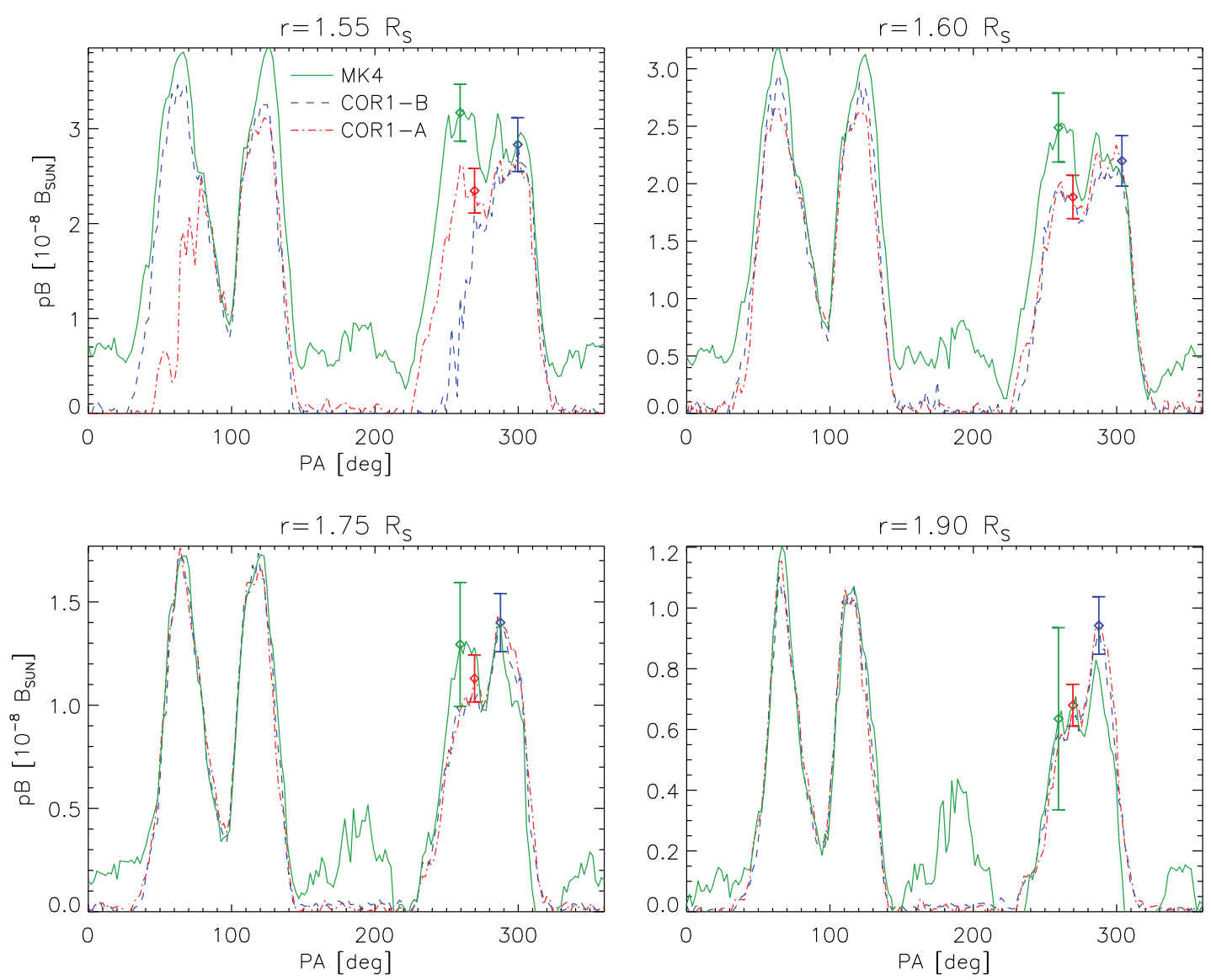

Figure 4 Comparison of the polarized brightness $[p B]$ profiles between Mk4 and COR1 at 1.55, 1.6, 1.75, and $1.9 R_{\odot}$. The error bars reflect the uncertainties detailed in Section 2.

\subsection{Mk4 and COR1}

The Mk4 and COR1 images can be compared over a substantial height range, from about 1.5 to $2.5 R_{\odot}$. A similar comparison between these two instruments is shown by Thompson et al. (2010), where two Mk4 plate scales were introduced, 5.81 and $6.00 \operatorname{arcsec~pixel}^{-1}$, but here we adopt the newly refined value of $5.785 \mathrm{arcsec}_{\mathrm{pixel}}^{-1}$. In addition, we applied a counter-clockwise rotation of $2.85^{\circ}$ (this corresponds to the value of the CROTA2 keyword in the FITS standard) to the Mk4 data (Thompson et al. (2010) applied a rotation of $2.5^{\circ}$ in their analysis).

Figure 3 displays the ratio of the COR $1 p B$ images to that of Mk4. This ratio image, and all subsequent ones, have been truncated at 0.0 and 2.0, and the white pixels correspond to values exceeding the upper limit. The polar values of this ratio are mostly near zero due to the COR1 background-subtraction method, but in the streamers below heights of about 2 $R_{\odot}$, the ratio is near unity. Figure 4 compares the COR 1 and Mk4 profiles extracted at 1.55 , $1.6,1.75$, and $1.9 R_{\odot}$, as a function of position angle [PA].

COR1 clearly does not perform well at $1.55 R_{\odot}$ at some position angles due to interference from the occulter. At $1.6 R_{\odot}$, all of the instruments agree to within the uncertainties over the east streamers, and agree, or come close to doing so, over most of the west streamer. The southern part of the west streamer is more problematic, likely due to interference from the occulter. At the other heights 1.75 and $1.90 R_{\odot}$, the agreement is almost perfect for the east streamers, whereas some discrepancy persists between the two nearly identical COR1-A 

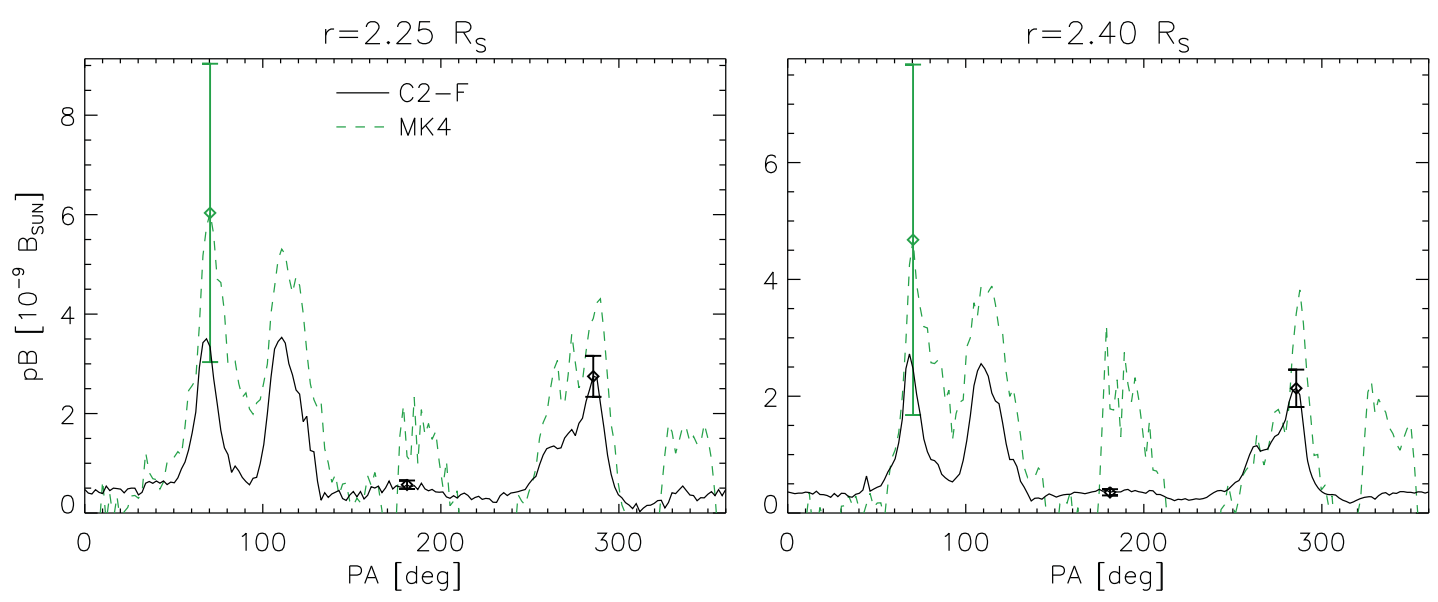

Figure 5 Comparison of the polarized brightness $[p B]$ profiles between $\mathrm{C} 2$ and Mk4 at 2.25 and $2.40 R_{\odot}$. The error bars reflect the uncertainties detailed in Section 2.

and COR1-B profiles and that of Mk4 for the west streamer, with the largest difference being well within the error bars. In the polar regions at all heights, the background-subtraction brings the COR1 values to near zero, whereas sky background and detector bit error strongly affect the Mk4 data.

\section{2. $\mathrm{C} 2$ and $\mathrm{Mk} 4$}

There is a very small range of heights over which both $\mathrm{C} 2$ and Mk4 obtain useful data, and there only in the brightest structures. The $\mathrm{C} 2 \mathrm{pB}$ data are compromised by the occulter below about $2.25 R_{\odot}$ (the $B$ images are good only above about $2.5 R_{\odot}$, due to the unpolarized diffraction rings around the image of the occulter), and the Mk4 uncertainty, mostly due to uncorrected sky polarization and the detector bit error, overwhelms the signal above $2.5 R_{\odot}$, even in the brightest of structures. Figure 5 compares $\mathrm{C} 2$ and Mk4 at 2.25 and $2.4 R_{\odot}$ as a function of PA. The Mk4 error bar half-width of $3 \times 10^{-9} B_{\odot}$ seems a bit too large, as a smaller correction would bring it into agreement with $\mathrm{C} 2$. The two instruments agree to within the stated uncertainties.

\section{3. $\mathrm{C} 2$ and $\mathrm{COR} 1$}

Figure 6 shows the ratio of the COR1 $p B$ images to those of $\mathrm{C} 2$. These ratio images have been limited at 0.0 and 2.0, and the white pixels correspond to values exceeding the upper limit. The image has been made black within $2.2 R_{\odot}$ to reflect the usable field of view of $\mathrm{C} 2$. The deficiencies of the COR1 background-subtraction method are clearly evident, as the ratio is close to zero in the polar regions.

Figure 7 compares the $\mathrm{C} 2$ and $\mathrm{COR} 1 p B$ profiles at $2.3,2.8,3.3$, and $3.8 R_{\odot}$, as a function of PA. COR1-A and -B show a very high degree of consistency, with their curves overlapping at most PAs. The largest discrepancy, about $12 \%$, takes place in the northeast streamer at the peak. However, this difference is well described by the $10^{-10} B_{\odot}$ background uncertainty estimate (see Section 2). In addition, COR1-A and -B disagree on the PA of the peak intensity of this streamer at $3.8 R_{\odot}$ by several degrees, most likely due to background-subtraction difficulties. The photon-counting noise is particularly evident at 3.3 and $3.8 R_{\odot}$, especially in COR1-A. The COR1 data are higher than $\mathrm{C} 2$ for all streamers; however, the error bars account for this difference. Despite this, the mean 
Figure 6 The ratio of the polarized brightness $[p B]$ COR1/C2 for COR1-A (top) and COR1-B (bottom). The white pixels correspond to values greater than the limits of the color scale. The radius of the blocked portion is $2.2 R_{\odot}$ (somewhat larger than the $\mathrm{C} 2$ occulter), and the overlaid grid starts at $2.2 R_{\odot}$, has circles every $0.5 R_{\odot}$ and radial lines every $30^{\circ}$.
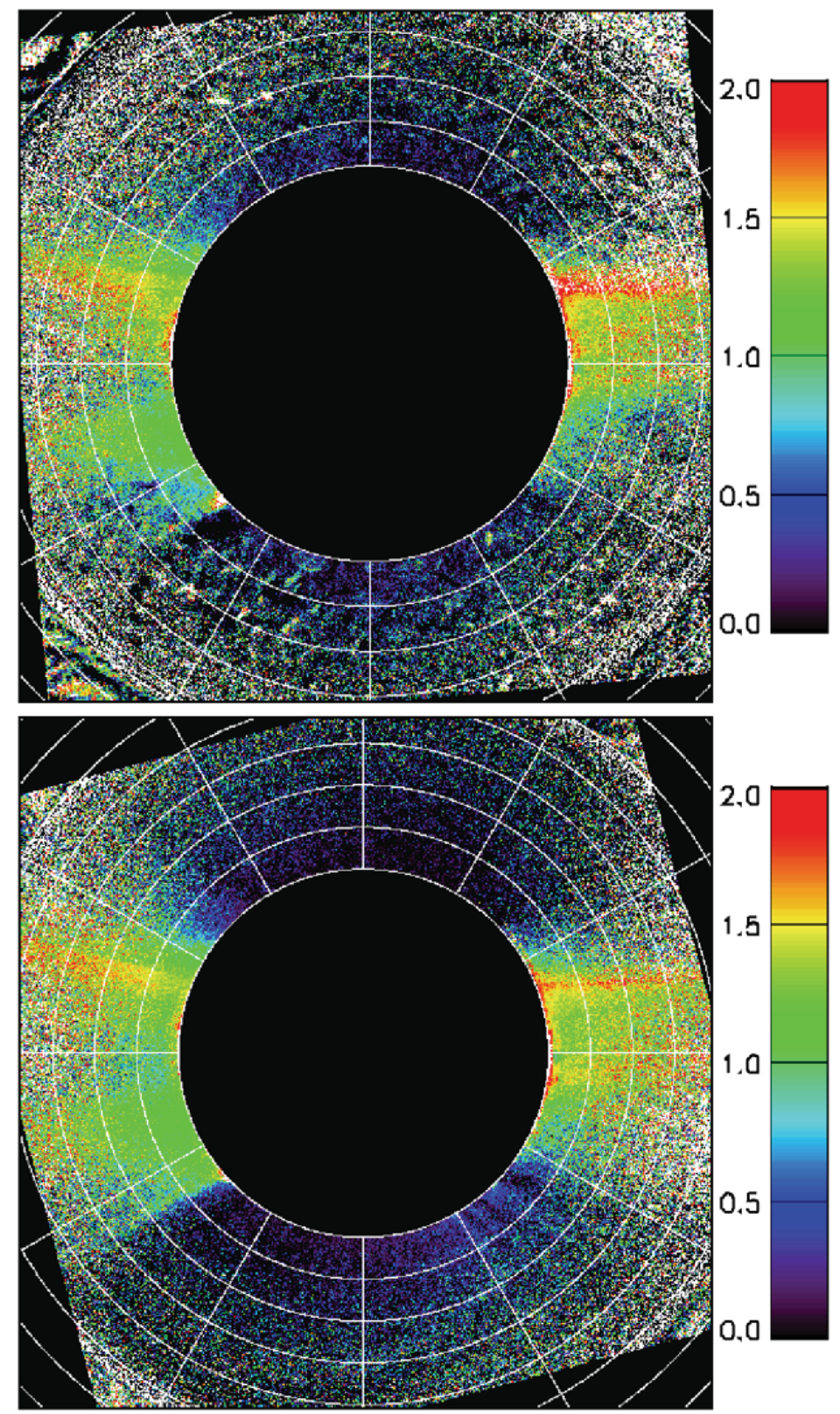

ratios between COR1 and $\mathrm{C} 2$, shown in Table 2, are very close to unity, due to the nonstreamer regions where $\mathrm{C} 2$ has a higher signal. The histograms in Figure 12 corresponding to COR1 and $\mathrm{C} 2$ show distributions peaked above unity, which correspond to the streamer regions, as well as a significant population below unity, corresponding to other regions. Note that Table 2 and Figure 12 excluded data within $30^{\circ}$ of the polar directions.

Figure 8 is similar to Figure 7, except that it compares the $B$ images. COR $1 B$ images are more sensitive to small errors in the background subtraction than $p B$ images, because the instrumental background is mostly unpolarized and therefore is largely removed by the $p B$ calculation. The large instrumental background is caused by COR1's internally occulted design, and the sensitivity of the background to the varying orbital distance causes the COR $1 B$ values to be less reliable in the fainter parts of the corona. In addition, there is no explicit re- 

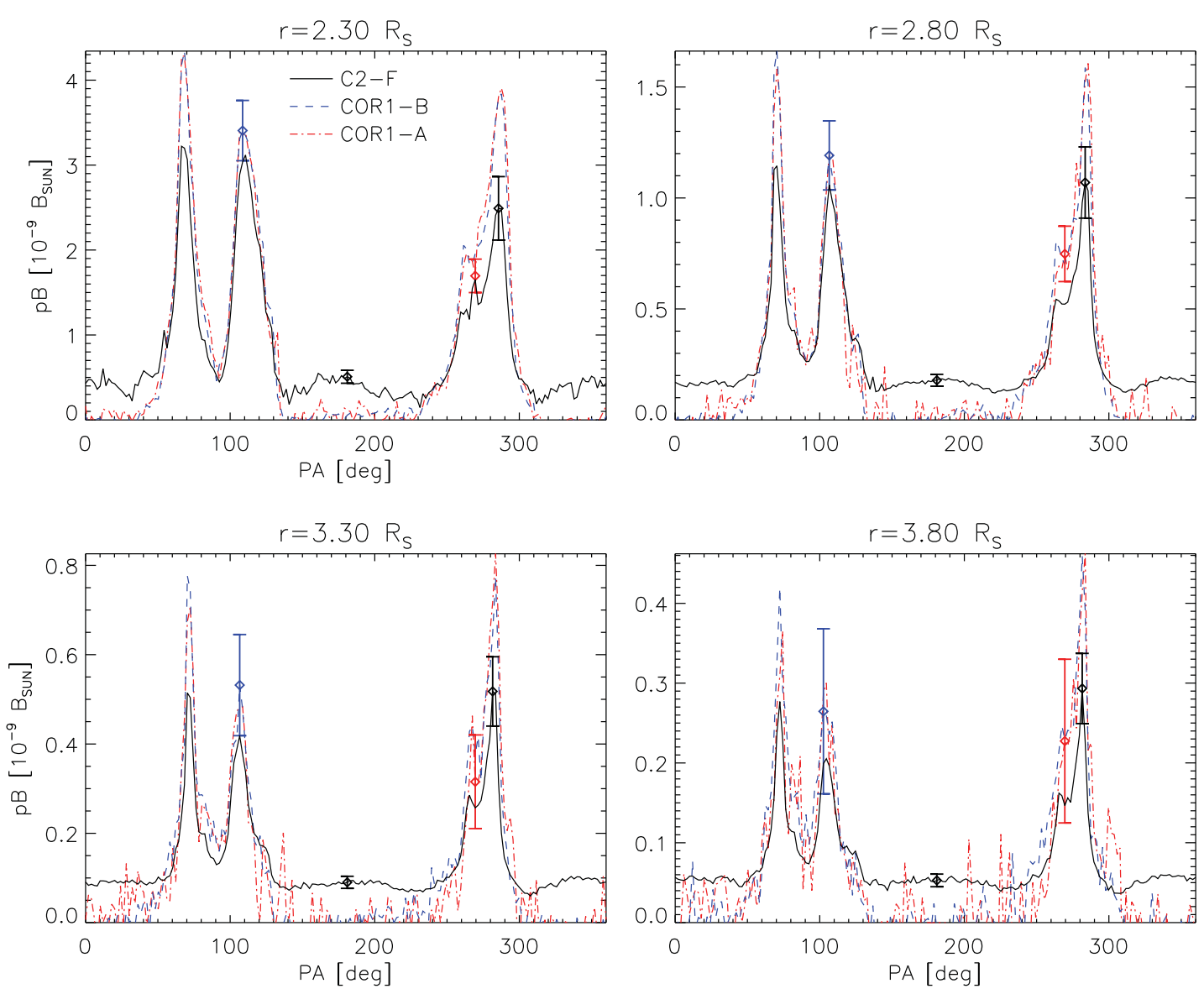

Figure 7 Comparison of the polarized brightness $[p B]$ profiles between $\mathrm{C} 2$ and COR 1 at 2.3, 2.8, 3.3, and $3.8 R_{\odot}$. The error bars reflect the uncertainties detailed in Section 2.

Table 2 Statistical comparison in overlap region of instruments, excluding $30^{\circ}$ on either side of each pole. For each instrument pair, the mean of the pixel ratios as well as the skewness and kurtosis of the pixel-ratio histogram (see Figure 12) are shown.

\begin{tabular}{llllllll}
\hline$I_{\alpha}$ & $I_{\beta}$ & Type & $\begin{array}{l}\text { Altitude } \\
{\left[R_{\odot}\right]}\end{array}$ & $\overline{I_{\alpha} / I_{\beta}}$ & Std. dev. & Skewness & Kurtosis \\
\hline Mk4 & $\mathrm{C} 2-\mathrm{F}$ & $p B$ & $2.25-2.4$ & 1.78 & 0.95 & 0.77 & 0.62 \\
COR1-A & $\mathrm{Mk4}$ & $p B$ & $1.6-1.9$ & 0.91 & 0.42 & 2.51 & 17.44 \\
COR1-B & Mk4 & $p B$ & $1.6-1.9$ & 0.90 & 0.40 & 2.99 & 22.91 \\
COR1-A & $\mathrm{C} 2-\mathrm{F}$ & $p B$ & $2.5-3.8$ & 1.04 & 0.56 & 0.47 & 0.93 \\
COR1-B & $\mathrm{C} 2-\mathrm{F}$ & $p B$ & $2.5-3.8$ & 0.96 & 0.51 & -0.12 & 0.05 \\
COR2-A & $\mathrm{C} 2-\mathrm{F}$ & $p B$ & $4.5-5.95$ & 0.57 & 0.25 & 1.71 & 8.11 \\
COR2-B & $\mathrm{C} 2-\mathrm{F}$ & $p B$ & $4.5-5.95$ & 1.19 & 0.39 & 0.94 & 1.83 \\
COR2-A & $\mathrm{C} 2-\mathrm{F}$ & $B$ & $4.5-5.95$ & 0.63 & 0.37 & 0.27 & 1.25 \\
COR2-B & $\mathrm{C} 2-\mathrm{F}$ & $B$ & $4.5-5.95$ & 1.07 & 0.56 & 0.71 & 0.53 \\
\hline
\end{tabular}

moval of the F-corona contribution in the COR1 data reduction (the background-subtraction procedure removes most of the F-corona contribution), unlike $\mathrm{C} 2$. The uncertainty estimates are not adequate to explain the differences in the $B$ values at most locations. 

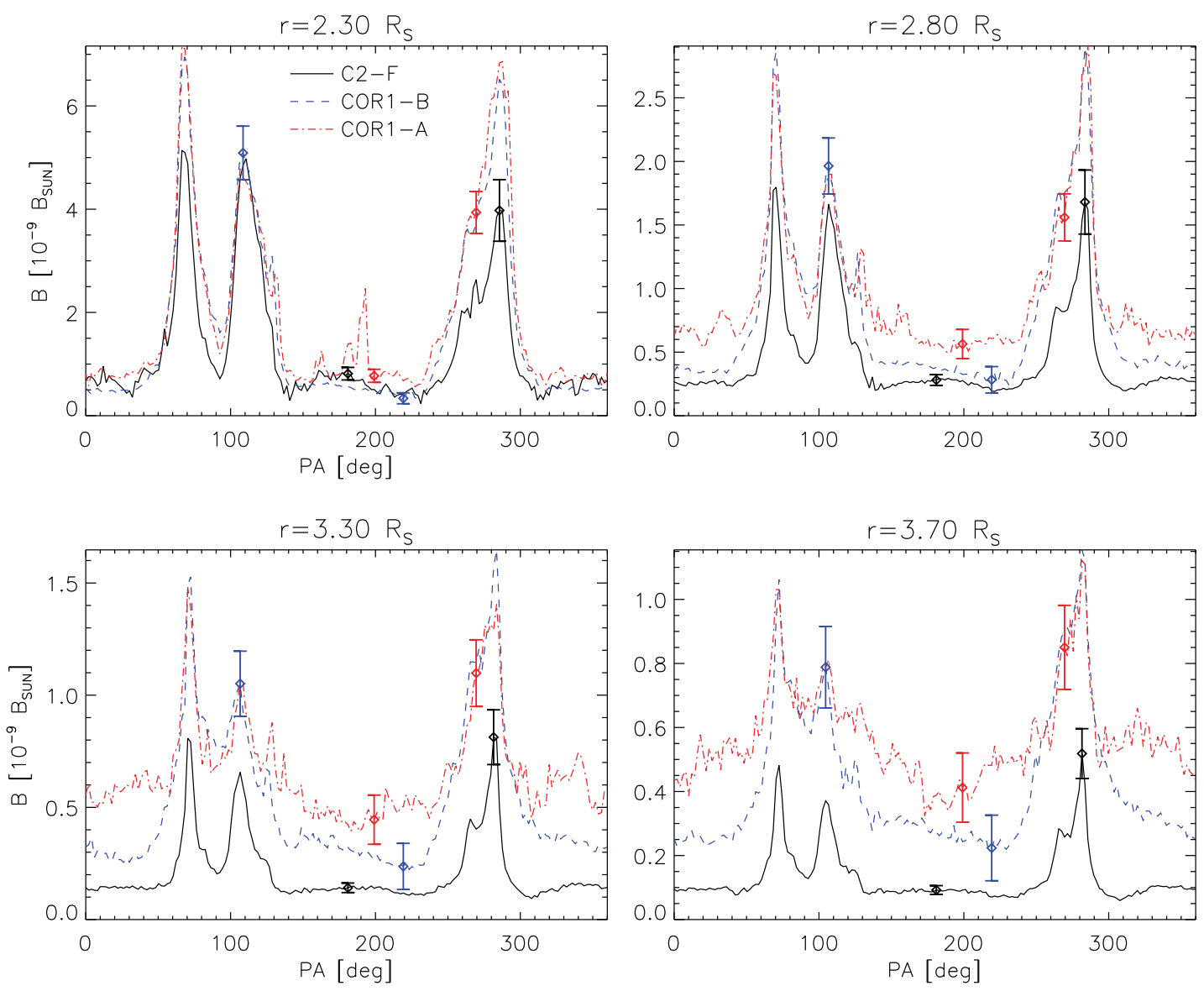

Figure 8 Comparison of the total brightness $[B]$ profiles between $\mathrm{C} 2$ and COR 1 at 2.3, 2.8, 3.3, and $3.7 R_{\odot}$. The error bars reflect the uncertainties detailed in Section 2.

\section{4. $\mathrm{C} 2$ and $\mathrm{COR} 2$}

Figure 9 shows the ratio of the COR $2 p B$ images to those of $C 2$, similarly to Figure 6 . These ratio images have been limited at 0.0 and 2.0 for display, and the white pixels correspond to values exceeding the upper limit. The figure shows that COR2-A and -B have significantly different properties which are more readily understood with the help of Figure 10, which compares the $\mathrm{C} 2$ and $\mathrm{COR} 2$ profiles at $4.5,5,5.5$, and $6 R_{\odot}$. Note that in these plots, $\mathrm{C} 2$ shows reasonable agreement with COR2-A (to within the uncertainties) only in the bright streamer structures near $\mathrm{PA}=70^{\circ}, 110^{\circ}$, and $280^{\circ}$, and that at all other PAs, the COR2-A signal is far below that of $\mathrm{C} 2$. Most of the discrepancy between COR2-A and -C2 cannot be explained by the given uncertainties, but we stress that the COR 2 error bar reflects only the $\approx 20 \%$ radiometric uncertainty and is a lower bound of the true, but unknown, uncertainty. The upper panel of Figure 9 shows exactly this trend with colors corresponding to ratios between about 0.8 and 1.5 (blue-green to yellow-green) at these same PAs, but dark-blue to violet almost everywhere else. Except for the white region near the center, indicating a large amount of polarized stray light, much of the lower panel of Figure 9 corresponds to ratios between about 0.9 and 1.5, indicating some global agreement between $\mathrm{C} 2$ and COR2B. Figure 10 further shows that COR2-B and C2 agree well in both the east streamers and polar regions, with the largest inconsistencies occurring near the northeast, southwest, and northwest limbs, especially above $5.0 R_{\odot}$. Besides these regions, the discrepancies between $\mathrm{C} 2$ and COR2-B are within the stated uncertainties. 
Figure 9 The ratio of the polarized brightness $[p B]$ COR2/C2 for COR2-A (top) and COR2-B (bottom). The white pixels correspond to values greater than the limit of the color scale. The radius of the blocked portion is $2.5 R_{\odot}$, and the overlaid grid has circles every $0.5 R_{\odot}$ and every $30^{\circ}$.
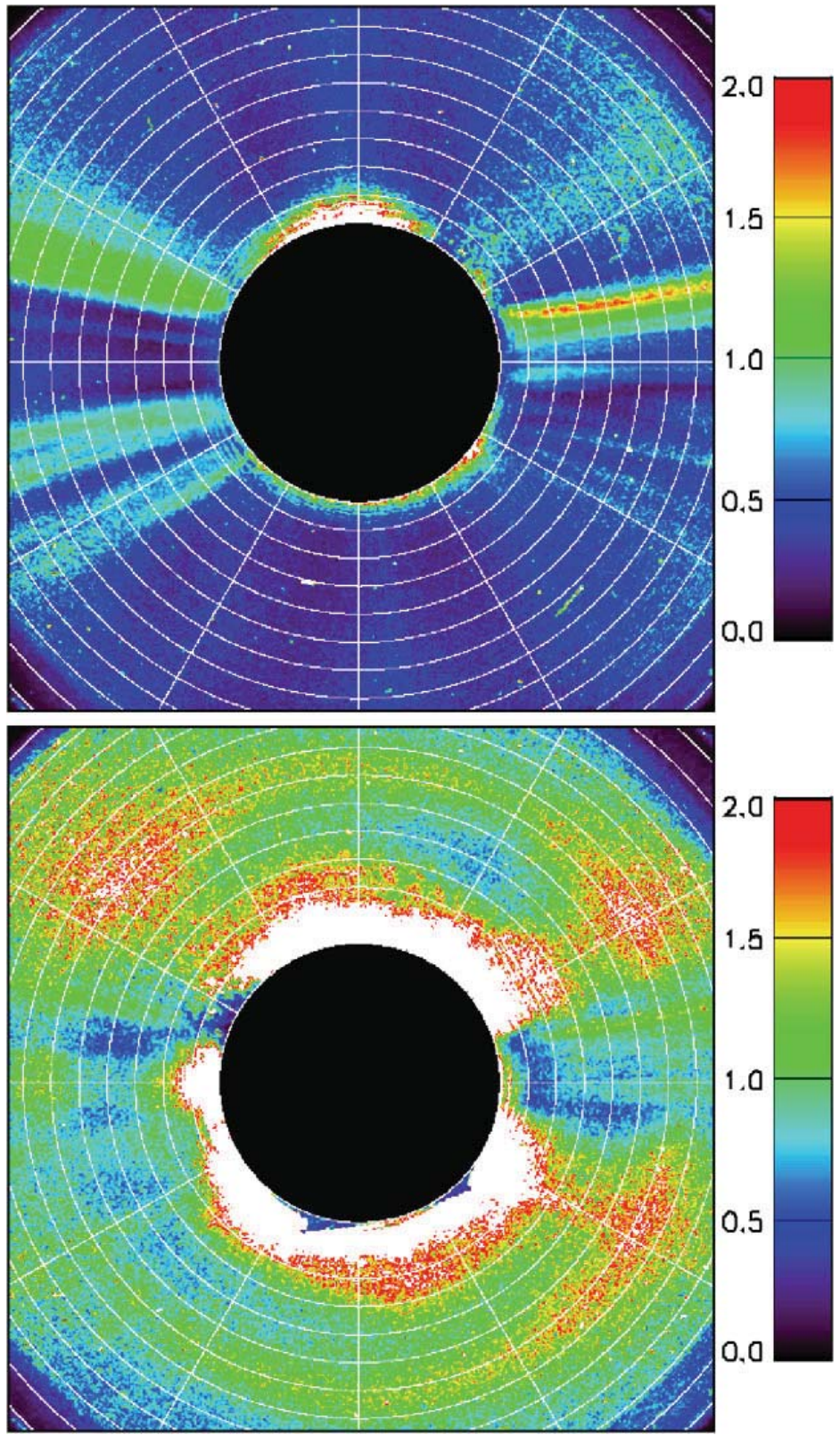

Images of the COR2-to-C2 total brightness $[B]$ ratio are quite similar to those displayed in Figure 9 and are not displayed here, but four profiles are shown in Figure 11, similarly to Figure 10. As is the case with COR1, the background-subtraction procedure removes much of the unpolarized stray light as well as some of the F-corona. COR2-A agrees with C2 in the brightest streamers near PA $=70^{\circ}$ and $270^{\circ}$, but almost nowhere else, and the error bars are not adequate to explain the differences. COR2-B agrees with $\mathrm{C} 2$ in the streamer near PA $=70^{\circ}$ and over the North Pole. As with the $p B$ images, there is a large uncorrected stray-light contribution in the Southwest of COR2-B, that even prevents agreement in the streamer near $\mathrm{PA}=280^{\circ}$.

At the moment it is difficult to explain the origins of the differences between COR2-A and $-\mathrm{B}$ and the generally superior agreement between COR2-B and $\mathrm{C} 2$ than is found between COR2-A and C2. Particularly vexing is the fact that COR2-B has a higher stray-light background than COR2-A with a polarized component (as shown by Figures 10 and 11). 

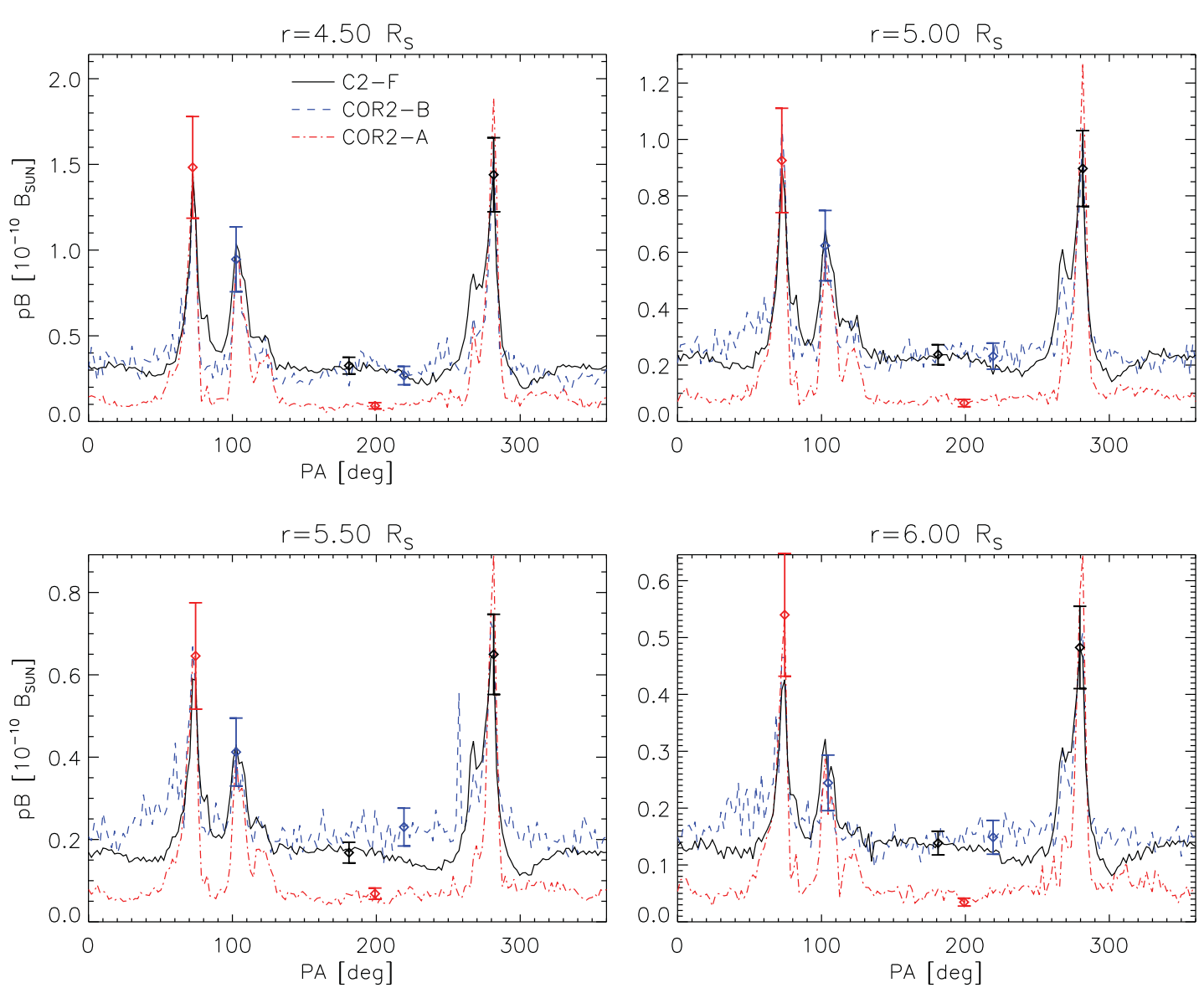

Figure 10 Comparison of the polarized brightness $[p B]$ profiles between $\mathrm{C} 2$ and COR2 at 4.5, 5.0, 5.5, and $6.0 R_{\odot}$. The error bars reflect the uncertainties detailed in Section 2.

In COR2-B, the polarized stray light is concentrated within a radius of $4 R_{\odot}$, while the unpolarized stray light is less localized. The COR2-B images are the results of a 2.0 second exposure compared to the 6.0 second exposure for COR2-A. Therefore, the photon noise in COR2-B is increased by about $\sqrt{3}$ compared to COR2-A. The reduced exposure will result in a reduced signal-to-noise ratio and less signal in the faint structures. In addition, COR2-A and $-\mathrm{B}$ have their occulter pylons at $\mathrm{PA}=315^{\circ}$ and $45^{\circ}$, respectively (that of $\mathrm{C} 2$ is at $\left.135^{\circ}\right)$. In COR2, the signal-to-noise ratio is lower at the pylon location due to vignetting (this is not an important effect in C2). Hence, the image ratios will be affected at those locations, as readily seen in the top panel of Figure 9. We note that some of the differences in Figures 9-11 seem to arise at the edges of streamers for COR2-A. This is likely due to streamer structure in the monthly minimum background. The superior agreement to $\mathrm{C} 2$ shown by COR2-B, despite the higher stray-light level, may be a fortuitous combination of systematic effects, especially partially polarized stray light, and the background-subtraction procedure. Further comparisons might elucidate the situation. The $180^{\circ}$ opposition in February 2011 affords the opportunity to compare COR2-A and -B to each other, as was done with COR1 (Thompson et al., 2011), but this has not yet been done quantitatively. In 2015, both of the STEREO spacecraft will be nearly in opposition to the Earth; however, STEREO observations will be limited in this period due to solar radio interference. 

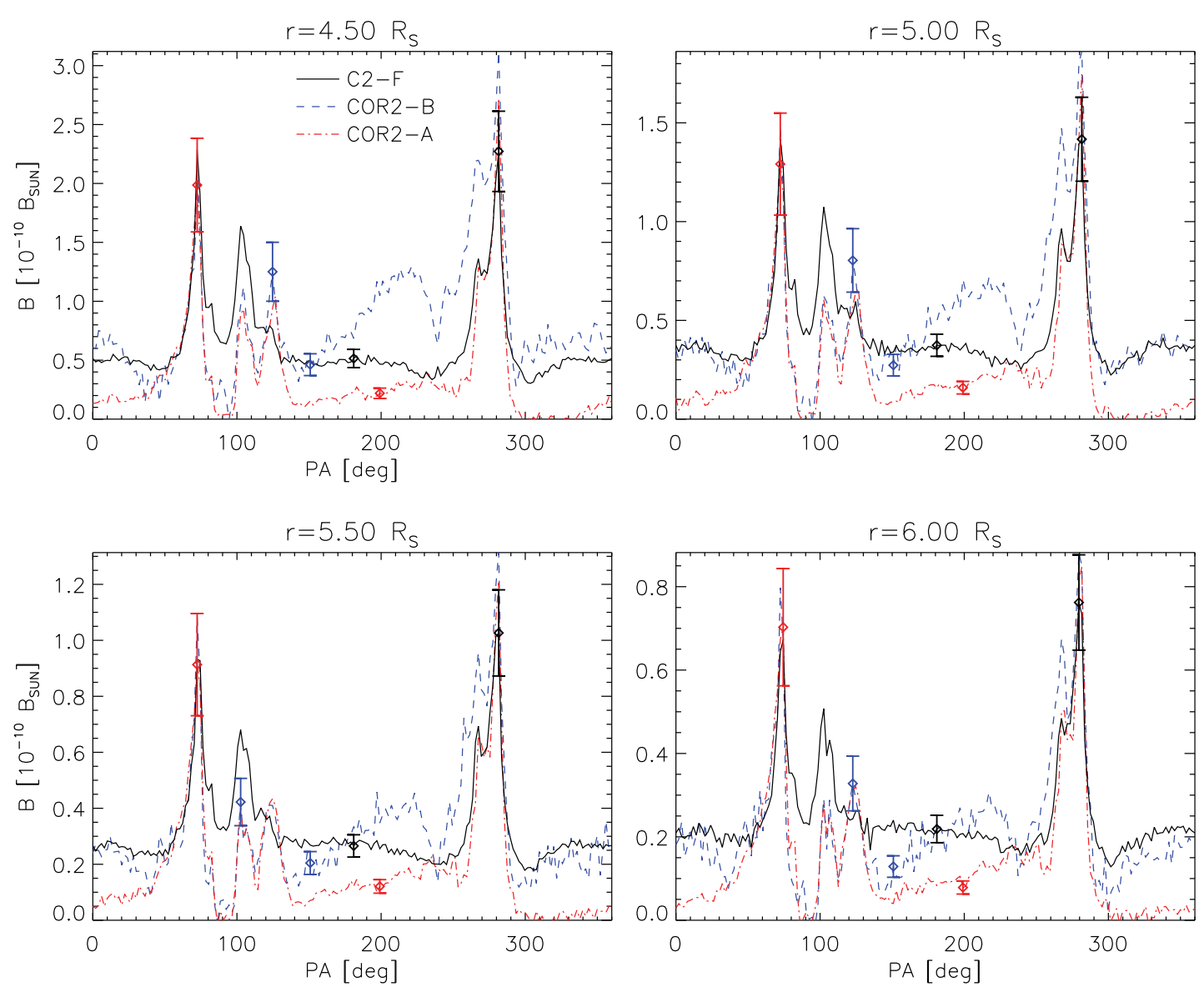

Figure 11 Comparison of the total brightness $[B]$ profiles between $\mathrm{C} 2$ and COR2 at 4.5, 5.0, 5.5, and $6.0 R_{\odot}$. The error bars reflect the uncertainties detailed in Section 2.

\subsection{Comparison Summary Statistics}

Table 2 summarizes the individual intercomparisons described in the previous sections. A given row of the table characterizes the spatial statistics of the ratio between two images $I_{\alpha} / I_{\beta}$, where $I_{\alpha}$ and $I_{\beta}$ are two images. The images are binned to nearly the same plate scale, so Mk4 is binned by a factor of four, COR1 by a factor of four, and COR2 by a factor of two; C2 is not binned. Image regions corresponding to $30^{\circ}$ on either side of each polar direction are excluded, because Mk4 has very weak polar signals at the comparison heights, and the COR1 signals over the poles are mostly removed by the background subtraction. Ratio values above 5.0, which account for at most $2.7 \%$ of compared pixels, as well as negative ratio values, are considered outliers and excluded in this analysis. For each instrument pair, Table 2 displays the mean of the pixel ratios, as well as the skewness and kurtosis of its histogram. The skewness and kurtosis are related to the third and fourth moments and tend toward zero for nearly normal distributions; therefore, these relatively large values of the skewness and kurtosis indicate that the ratio histograms are not close to normal. This non-normality is further illustrated in Figure 12, which shows the histograms of the various pixel ratios. The histograms are divided by the population size, so adding the population of any histogram yields unity. For illustration, superimposed on each histogram is a Gaussian curve with the same mean and variance (see Table 2) as the histogram, and it is normalized so that it integrates to unity. 

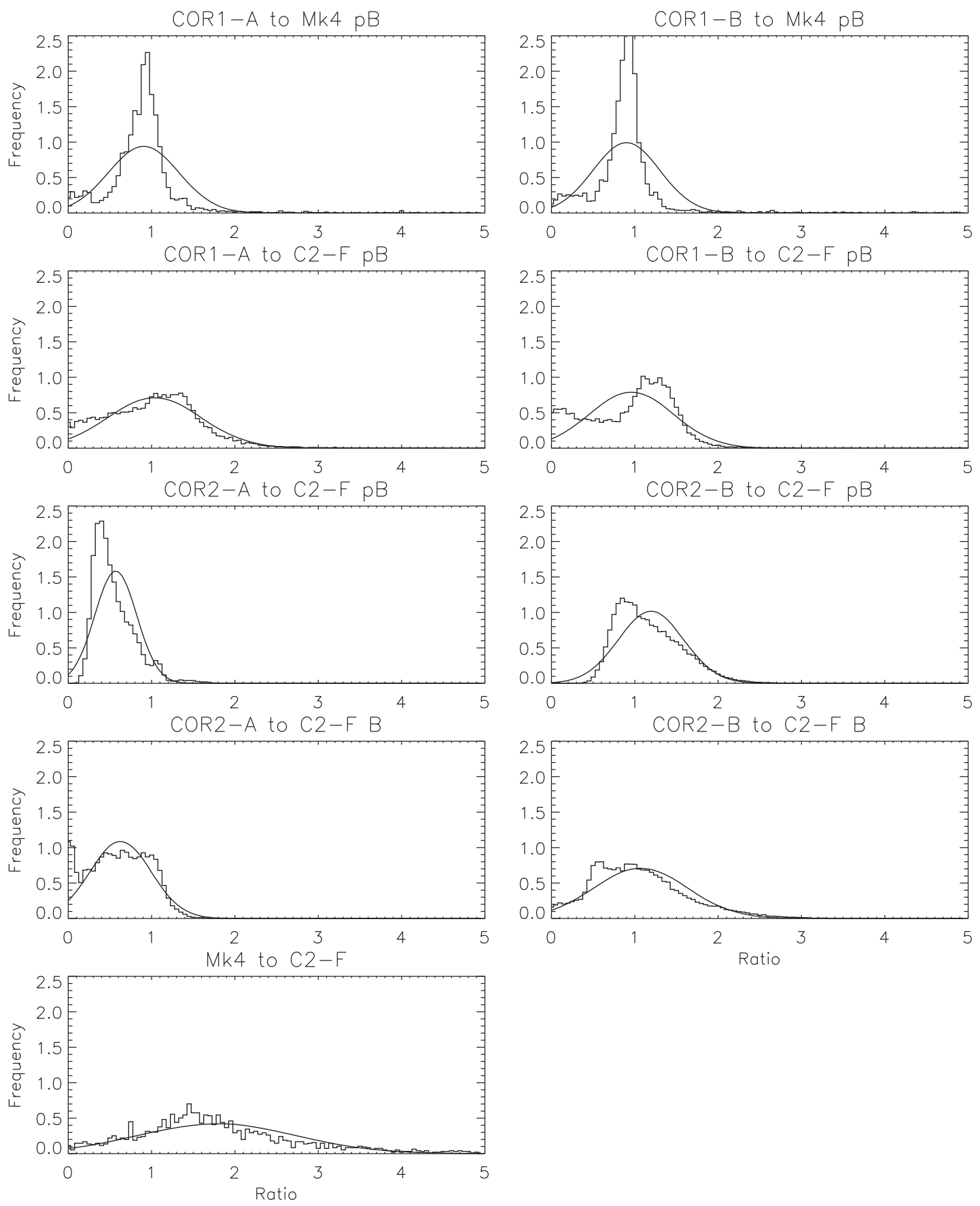

Figure 12 Normalized histograms of the pixel ratios for the different comparisons performed in this study. A Gaussian distribution with the same area, mean, and standard deviation is shown for comparison.

\section{Discussion}

Given the astrophysical importance of reliable $p B$ and $B$ measurements of the K-corona, it is important to compare white-light coronagraphs for consistency. Many of the difficulties found here in comparing data between coronagraphs relate to the problems associated with background subtraction. Since the brightest CMEs give stronger signals than the streamers analyzed here, the difficulties associated with background subtraction are unlikely to play a major role, and all of these instruments should provide consistent and reliable intensities for 
these events (assuming the CME does not change too much during a polarization sequence). For less bright structures (e.g. coronal holes), accurate background subtraction is vital for applications that require accurate absolute intensities, such as electron-density determination (Hayes, Vourlidas, and Howard, 2001; Quémerais and Lamy, 2002; Frazin et al., 2010). Other uses of coronagraph data depend only upon differences in brightness. For example, one might track transients with running-difference images (Rouillard et al., 2011) or analyze images of CMEs by subtracting a pre-event image (Moran, Davila, and Thompson, 2010; Vourlidas et al., 2010), and the conclusions drawn here about the comparisons between telescopes may not be relevant in such cases.

This study has emphasized comparing $\mathrm{C} 2$ to the other coronagraphs. This implicitly assumes that the $\mathrm{C} 2$ calibration made by the LAM team is the most reliable and therefore is the standard to which the others should be compared. This approach is justified for several reasons.

- $\mathrm{C} 2$ has avoided some of the problems that arise in Mk4, COR1, and COR2:

- C2 is in space and has no problems with polarized sky background or other atmospheric phenomena, unlike Mk4.

- C2 has external occultation, which prevents most light from the solar disk from entering the instrument in the first place. Note that the COR1 mission objective of observing close to the limb precluded external occultation designs, due to vignetting issues.

- C2 has a mechanism to center its inner occulter that allowed readjustment after launch stress to minimize stray light. COR1 and COR2 do not.

- C2 has the capacity to take images with no polarizer sheets in the optical path. This provides information about the imperfections of the polarizers. COR 1 and COR2 do not have this capability.

- The final calibration of $\mathrm{C} 2$ is the result of an extensive 17-year effort involving detailed analysis of the instrument, using many different data sources ranging from pre-flight laboratory measurements to stellar observations. This work will be given a thorough description in an upcoming publication by the LAM team.

- Analysis of $p B$ measurements made by ground-based telescopes during eclipses most notably, the Planetary Origins Imaging Spectrograph (POISE) measurements from 26 February 1998 - show excellent quantitative agreement to C2-F, both in coronal holes and streamers. This work will also be the subject of a forthcoming publication.

There is no simple way to characterize the differences seen between the various coronagraph measurements, but several general features stand out.

- The C2-F (LAM calibration) and C2-P (initial calibration) $p B$ values are different, and the community should adopt the LAM calibration as soon as it is made publicly available. Until then, a first-order correction of the C2-P $p B$ values would be to divide the images generated with the initial calibration by 1.50 .

- Mk4 is most accurate below $1.5 R_{\odot}$, but the overlap with the effective field of view of the COR 1 instruments begins at about $1.55 R_{\odot}$, where the influence of sky polarization is significant but not necessarily dominant. $\mathrm{C} 2$ and $\mathrm{Mk} 4$ can only be compared between about 2.25 and $2.4 R_{\odot}$, where the Mk4 values are highly uncertain. Due to the large uncertainty of Mk4 beyond $1.5 R_{\odot}$, it was found that it agrees, or nearly so, with both COR 1 and C2 everywhere. Overall, the comparisons of Mk4 with COR1 and C2 indicate that Mk4 provides accurate $p B$ values in bright streamers up to about $2.0 R_{\odot}$.

- The COR1 background procedure is inadequate for coronal-hole $p B$ measurement at all heights and for $B$ values above about $2.3 R_{\odot}$. However, the backgrounds do produce a high degree of consistency between the A and B spacecraft in the streamers. Since A and 
B have very different stray-light properties, this is a strong validation of the backgroundsubtraction methodology.

- At the streamer latitudes, and at all heights, the $\mathrm{C} 2$ and COR1 $p B$ values agree to within the stated uncertainties, but the COR1 values are systematically higher.

- At most position angles, COR2-B $p B$ values are consistent with $\mathrm{C} 2$, while those of COR2A are only consistent in bright structures. There is little consistency between the $B$ values of COR2 and $\mathrm{C} 2$.

In the streamer regions, we have found that $\mathrm{Mk} 4, \mathrm{COR} 1, \mathrm{C} 2$, and COR2 generally have consistent $p B$ values, which validates the calibrations and data-reduction procedures of all of these instruments to a significant degree. Due to COR1 background-subtraction issues, this study was unable to validate Mk4 polar $p B$ values, but the polar $p B$ values of $C 2$ and COR2-B were fairly consistent. This study underscores the need for additional validation measures, such as comparisons to eclipses or rocket flights. A comparison of the $\mathrm{C} 2$ and POISE measurements of the 26 February 1998 eclipse has yielded excellent results, but it has not yet been published. In the future, it would be useful to revisit the comparison with updated calibration procedures. Since the coronagraphs exhibit variable degrees of inconsistency, any applications that combine them, such as multi-spacecraft tomography (Frazin, Vásquez, and Kamalabadi, 2009), must analyze the effect that this inconsistency has on the results.

\subsection{Recommendations for Future Instrumentation}

This article closes with some lessons learned from the experience with the instruments considered here. It is hoped that this exercise will prove valuable for those planning future space-based coronagraphs. Ground-based coronagraphs are limited by sky background and thus present a different set of problems. Space-based systems may be limited by stray light (which may be elliptically polarized), unforeseen effects in hardware components in the optical train, or, for $B$ measurements, the ability to subtract the F-corona. Some of the items listed below have not been discussed here, but the instrument teams feel that they are important.

- Folded designs employing mirrors working at $45^{\circ}$, as in $\mathrm{C} 2$, should be avoided. This creates retardance, resulting in the "X" pattern discussed in Section 1.

- Polaroid foil (used in LASCO) should be avoided, and polarizers from a new generation (metallic coating) should be considered.

- The method of a single rotating polarizer (used by COR1 and COR2) is likely to provide easier in-flight calibration than LASCO's method of using three separate polarizers. Note that the former provides the helpful capability of measuring the polarization at any angle. Taking some synoptic data with four or more angles should be considered.

- The capacity to remove polarization elements from the optical train is helpful for consistency checks and calibration.

- Mechanisms for in-flight calibration of the polarizers would be quite helpful.

- Satellite rolls are important for polarization calibration.

- A synoptic sequence containing some losslessly compressed images would improve the process of separating the F- and K-coronae. The separation algorithm assumes that the F-corona does not have high-frequency features; thus the suppression of high-frequency components inherent in lossy compression is not helpful.

- Substantial overlap between instruments' fields of view is desirable for cross-calibration. LASCO-C2 and -C3 had a good compromise, but the smaller overlap between COR1 and COR2 may have been too ambitious. 
- The amount of stray light must be further reduced. Most importantly,

- The performance of external occulters needs to be improved.

- Internal adjustment of the internal and external occulters is required. (C2 has a mechanism for centering the internal occulter. We note that the COR2 coronagraphs are the first externally occulted instruments flown without an alignment mechanism, which adds mass, cost, and complexity.)

- The compromises between stray-light reduction, vignetting, and field-of-view limitation at each optical conjugation (e.g. the internal occulter is optically conjugate to the external occulter) must be carefully optimized.

Acknowledgements The authors thank the anonymous referee for helpful comments, which resulted in a much improved manuscript. This research was supported by NASA Heliophysics Guest Investigator award NNX08AJ09G to the University of Michigan. A.M.V. acknowledges UBACyT (Universidad de Buenos Aires) grant no. 20020090100264 for partial financial support. R.J.H. was funded by NASA under GSRP fellowship NNX08AT43H FLL. The LASCO-C2 project at Laboratoire d'Astrophysique de Marseille is funded by the Centre National d'Etudes Spatiales (CNES). The authors thank Katherine Baldwin for her helpful comments.

LASCO was built by a consortium of the Naval Research Laboratory, USA, the Laboratoire d'Astrophysique de Marseille (formerly Laboratoire d'Astronomie Spatiale), France, the Max-Planck-Institut für Sonnensystemforschung (formerly Max-Planck-Institut für Aeronomie), Germany, and the School of Physics and Astronomy, University of Birmingham, UK. SOHO is a project of joint collaboration by ESA and NASA.

The STEREO/SECCHI data used here are produced by an international consortium of the Naval Research Laboratory (USA), Lockheed Martin Solar and Astrophysics Lab (USA), NASA Goddard Space Flight Center (USA), Rutherford Appleton Laboratory (UK), University of Birmingham (UK), Max-Planck-Institut für Sonnensystemforschung (Germany), Centre Spatiale de Liège (Belgium), Institut d'Optique Théorique et Appliquée (France), and Institut d'Astrophysique Spatiale (France).

\section{References}

Brueckner, G.E., Howard, R.A., Koomen, M.J., Korendyke, C.M., Michels, D.J., Moses, J.D., et al.: 1995, Solar Phys. 162, 357. doi:10.1007/BF00733434.

Colaninno, R.C., Vourlidas, A.: 2009, Astrophys. J. 698, 852.

Elmore, D.F., Burkepile, J.T., Darnell, J.A., Lecsinki, A.R., Stanger, A.L.: 2003, SPIE 4843, 66.

Frazin, R.A., Romoli, M., Kohl, J.L., Gardner, L.D., Wang, D., Howard, R.A., Kucera, T.A.: 2002, In: Pauluhn, A., Huber, M.C.E., von Steiger, R. (eds.) The Radiometric Calibration of SOHO SR-2, ESA, Noordwijk, 249.

Frazin, R.A., Vásquez, A.M., Kamalabadi, F.: 2009, Astrophys. J. 701, 547.

Frazin, R.A., Lamy, P., Llebaria, A., Vásquez, A.M.: 2010, Solar Phys. 265, 19. doi:10.1007/s11207-0109557-9.

Frost, E.B.: 1900, Astrophys. J. 12, 307.

Hayes, A.P., Vourlidas, A., Howard, R.A.: 2001, Astrophys. J. 548, 1081.

Howard, R.A., Moses, J.D., Vourlidas, A., Newmark, J.S., Socker, D.G., Plunkett, S.P., et al.: 2008, Space Sci. Rev. 136, 67.

Jin, M., Manchester, W.B., van der Holst, B., Gruesbeck, J.R., Frazin, R.A., Landi, E., et al.: 2012, Astrophys. J. 745, 6. doi:10.1088/0004-637X/745/1/6.

Kohl, J.L., Esser, R., Gardner, L.D., Habbal, S., Daigneau, P.S., Dennis, E.F., Nystrom, G.U., Panasyuk, A., Raymond, J.C., Smith, P.L.: 1995, Solar Phys. 162, 313. doi:10.1007/BF00733433.

Llebaria, A., Lamy, P., Danjard, J.-F.: 2006, Icarus 182, 281.

Llebaria, A., Loirat, J., Lamy, P.L.: 2010, SPIE 7533, 75330.

Lee, K.-S., Moon, Y.-J., Kim, K.-S., Cho, K.-S., Choe, G.S.: 2008, Astron. Astrophys. 486, 1009.

Lyot, B.: 1939, Mon. Not. Roy. Astron. Soc. 99, 580.

Moran, T.G., Davila, J.M., Morrill, J.S., Wang, D., Howard, R.A.: 2006, Solar Phys. 237, 211. doi:10.1007/ s11207-006-0147-9.

Moran, T.G., Davila, J.M., Thompson, W.T.: 2010, Astrophys. J. 712, 453.

Morgan, H., Habbal, S.R.: 2007, Astron. Astrophys. 471, L47.

Morgan, H., Habbal, S.R., Woo, R.: 2006, Solar Phys. 236, 263. doi:10.1007/s11207-006-0113-6. 
Romoli, M., Frazin, R.A., Kohl, J.L., Gardner, L.D., Cranmer, S.R., Reardon, K., Fineschi, S.: 2002, In: Pauluhn, A., Huber, M.C.E., von Steiger, R. (eds.) The Radiometric Calibration of SOHO SR-2, ESA, Noordwijk, 181.

Rouillard, A.P., Sheeley, N.R. Jr., Cooper, T.J., Davies, J.A., Lavraud, B., Kilpua, E.K.J., et al.: 2011, Astrophys. J. 734, 7.

Thompson, W.T.: 2006, Astron. Astrophys. 449, 791.

Thompson, W.T., Reginald, N.L.: 2008, Solar Phys. 250, 443. doi:10.1007/s11207-008-9228-2.

Thompson, W.T., Wei, K., Burkepile, J.T., Davila, J.M., St. Cyr, O.C.: 2010, Solar Phys. 262, 213. doi:10. 1007/s11207-010-9513-8.

Thompson, W.T., Davila, J.M., St. Cyr, O.C., Reginald, N.L.: 2011, Solar Phys. 272, 215. doi:10.1007/ s11207-011-9815-5.

van de Hulst, H.C.: 1950, Bull. Astron. Inst. Neth. 11(410), 135.

Vourlidas, A., Howard, R.A., Esfandiari, E., Patsourakos, S., Yashiro, S., Michalek, G.: 2010, Astrophys. J. 722, 1522.

Zuccarello, F.P., Bemporad, A., Jacobs, C., Mierla, M., Poedts, S., Zuccarello, F.: 2012, Astrophys. J. 744, 66. doi:10.1088/0004-637X/744/1/66. 